\title{
L'identité religieuse affichée par l'État et ses conséquences sous surveillance
}

Gérard Gonzalez

\section{(2) OpenEdition}

1 Journals

Édition électronique

URL : https://journals.openedition.org/rdr/1229

DOI : $10.4000 /$ rdr.1229

ISSN : 2534-7462

Éditeur

Presses universitaires de Strasbourg

\section{Édition imprimée}

Date de publication : 16 novembre 2020

Pagination : 89-103

ISBN : 979-10-344-0073-7

ISSN : 2493-8637

Référence électronique

Gérard Gonzalez, «L'identité religieuse affichée par l'État et ses conséquences sous surveillance », Revue du droit des religions [En ligne], 10 | 2020, mis en ligne le 16 novembre 2020, consulté le 03 mai 2022. URL : http://journals.openedition.org/rdr/1229; DOI : https://doi.org/10.4000/rdr.1229

\section{(c) (†) (8)}

La revue du droit des religions est mise à disposition selon les termes de la Creative Commons Attribution - Pas d'Utilisation Commerciale 4.0 International - CC BY-NC 4.0. 


\section{L'IDENTITÉ RELIGIEUSE AFFICHÉE PAR L'ÉTAT ETSES CONSÉQUENCESSOUSSURVEILLANCE}

\section{Gérard GONZALEZ}

Université de Montpellier, Institut de droit européen des droits de l'homme (IDEDH)

Université de Strasbourg / CNRS, Droit, Religion, Entreprise et Société (DRES)

\section{RÉSUMÉ}

L'État a droit à une identité religieuse dont les racines plongent souvent dans les tréfonds de son histoire. Il peut afficher son attachement à cette identité, entretenir des relations privilégiées avec la religion dominante. Mais, dans une société vraiment démocratique, toute différence de traitement doit demeurer objective et raisonnable, respectueuse des valeurs du pluralisme et de la tolérance. L'abus de position dominante d'une confession n'est pas acceptable. La Cour européenne des droits de l'homme veille à cet équilibre globalement respecté dans les États membres du Conseil de l'Europe. Ailleurs, notamment dans les pays où l'islam est religion majoritaire, c'est plus difficile.

\section{Abstract}

The State is entitled to a religious identity whose roots often deeply lie in history. It can show his attachment to this identity, maintaining privileged relations with the dominant religion. But in a truly democratic society, any difference in treatment must remain objective and reasonable, respecting the values of pluralism and tolerance. The abuse of a dominant position by a denomination is not acceptable. The European Court of Human Rights ensures that this balance is generally respected in the member states of the Council of Europe. Elsewhere, especially in countries where Islam is the majority religion, it is more difficult. 
T a revendication identitaire n'est pas l'apanage des individus bien que, _déclinée à l'aune des convictions religieuses, elle se rapporte essentiellement à eux prima facie. On voudrait nous faire accroire que le temps de l'Histoire qui ancrait l'État, le souverain, la nation à une religion est révolu; que nous sommes sortis «d'un monde où la religion est structurante, où elle commande la forme politique des sociétés et où elle définit l'économie du lien social ${ }^{1} »$ ou «de l'identité domestique ${ }^{2} »$, celle qui fonde une nation sur divers marqueurs dont, historiquement, la religion fait partie. Il est un fait que l'État moderne, de préférence «de droit», est forcément converti au pluralisme, à la tolérance, à l'esprit d'ouverture et que le modèle laïque semble le plus compatible avec ces principes directeurs. De tous les systèmes connus de rapports entre l'État et la religion, la laïcité est celui qui a priori apparaît le plus propice à l'épanouissement de la liberté de religion par la distance maintenue entre le séculier et le religieux, garantie d'une neutralité cependant relative; il y a toujours dans un système laique une forme ou une autre de «reconnaissance» (qui ne dit pas son nom) de certaines religions; il suffit dans ce cas que les critères posés soient objectifs et non-discriminatoires $^{3}$. La Cour européenne des droits de l'homme considère d'ailleurs que le principe de laïcité est « respectueux des valeurs sous-jacentes à la Convention ${ }^{4} »$ ou encore, franchissant un palier, «conforme ${ }^{5}$ » à ces valeurs. Mais si tout État démocratique se doit de respecter le pluralisme religieux, il n'est pas besoin pour cela que lui-même soit totalement areligieux, laique au sens strict du terme ${ }^{6}$. Dans le monde, où la démocratie n'est souvent que de façade, nombreux sont les États, notamment arabo-musulmans, à accorder une place prépondérante à une religion. Nombreux sont encore les États qui affichent leur identité religieuse de diverses façons, plus ou moins directement (1). Cet affichage est l'objet de tensions notamment avec les minorités qui revendiquent le respect de leur propre identité; il doit être, dans une société démocratique et pluraliste, encadré (2).

1. M. GAuchet, La religion dans la démocratie, Paris, Gallimard, 1998, p. 13.

2. A. Finkielkraut, L'identité malheureuse, Paris, Stock, 2013, p. 104.

3. Par ex. les associations cultuelles de la loi de 1905 en France. Comme on a pu l'écrire, «le droit français concernant la liberté religieuse, la laïcité de l'État et sa neutralité ne s'est déterminé et ne se détermine encore qu'en fonction de la place qu'a en France l'Église catholique»(R. Drago, «Laïcité, neutralité, liberté?», Arch. phil. dr., Droit et religion, 1993, p. 222).

4. CEDH, Gde ch., 10 nov. 2005, Leyla Şahin c. Turquie, § 114.

5. CEDH, déc. 30 juin 2009, Aktas c. France.

6. C'est-à-dire séparé de toutes les religions, à distance de celles-ci. 


\section{L'IDENTITÉ RELIGIEUSE AFFICHÉE}

Cet affichage peut prendre plusieurs formes. La plus solennelle est l'affirmation constitutionnelle de l'identité religieuse d'État (1.1), mais il peut aussi être assumé par la mise en œuvre de politiques publiques ou de traditions plus ou moins ouvertement favorables à la religion dominante (1.2).

\subsection{UN ACTE DE FOI CONSTITUTIONNEL}

L'ensemble des pays dont la population est majoritairement musulmane se réfèrent à l'islam dans leur Constitution ${ }^{7}$, la charia constituant pour certains d'entre eux une source du droit ${ }^{8}$. Ces exemples sont connus et l'influence de l'islam se retrouve dans les organisations qui regroupent ces États ${ }^{9}$ et dans les textes qu'ils s'efforcent d'élaborer pour concurrencer les textes internationaux de protection des droits de l'homme trop marqués, selon eux, par l'identité judéo-chrétienne et occidentale ${ }^{10}$. Revenons à l'Europe.

7. À l'exception du Liban «arabe dans son identité et son apparence» prônant la «suppression du confessionnalisme politique» (préambule de la Constitution du 23 mai 1926) qui, tout de même «en rendant Hommage au Très Haut», affirme respecter et protéger toutes les confessions (art. 9), c'est le cas des pays arabo-musulmans (art. 3 de la Constitution du $1^{\text {er }}$ juillet 2011 du Maroc, art. 2 de la Constitution algérienne du 28 novembre 1996, art. 2 de la Constitution égyptienne de 2014, art. 2 de la Constitution jordanienne du

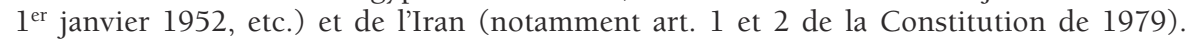
Pour l'Arabie saoudite: S. Dumartin Yaodia, «L'influence de l'Islam sur la Constitution: l'exemple de l'Arabie Saoudite», RFD const. 2020, p. 391-413.

8. L'Arabie Saoudite (art. 11 de la loi fondamentale de 1992), l'Égypte (art. 2), le Qatar (art. 1 Constitution du 29 avril 2003). Pour l'Iran: A. Amir-ARJOMAND, «La charia en Iran », in B. Dupret (dir.), La charia aujourd'hui, Paris, La Découverte, 2012, p. 171-184.

9. La Charte de l'OCI (Organisation de la coopération islamique) adoptée «au nom de Dieu, le Clément, le Miséricordieux» prévoit que peut adhérer «tout État membre des Nations Unies disposant d'une majorité musulmane» (art. 3). L'OCI doit «Propager, promouvoir et préserver les enseignements et les valeurs islamiques fondés sur la modération et la tolérance, promouvoir la culture islamique et sauvegarder le patrimoine islamique» (art. 1-11), «Protéger et défendre la véritable image de l'Islam, lutter contre la diffamation de l'Islam et encourager le dialogue entre les civilisations et les religions» (art. 1-12)...

10. Par ex. la Déclaration islamique universelle des droits de l'homme adoptée le 19 septembre 1981, la Charte arabe des droits de l'homme du 15 septembre 1994 révisée le 23 mai 2014. Comme l'écrit Mustapha Afroukh, «le champ d'application de ces textes se définit à partir d'un critère d'appartenance religieuse, à savoir l'appartenance à l'Umma islamique. C'est moins l'Homme qui est ici visé que le musulman» («Rapport introductif», in M. Afroukh (dir.), L'islam en droit international des droits de l'homme, Bayonne, Institut universitaire Varenne, 2019, p. 18). V. aussi pour une vision plus optimiste mais contestable, dans le même ouvrage: F. QuillerÉ-MAzJoub, «L'existence d'un système arabo-musulman des droits de l'homme», p. 219-233. Certains de ces États, minoritaires sont parties aux grands instruments internationaux de protection des droits de l'homme comme le Pacte 
Dans l'Union européenne, la question religieuse apparaît à la marge, pour la première fois, dans la déclaration $\mathrm{n}^{\circ} 11$ annexée au traité d'Amsterdam adopté le 2 octobre 1997 consacrant la préservation des statuts spécifiques des confessions reconnues «en vertu du droit national». Le débat lors de l'adoption de la Charte des droits fondamentaux en 2000 et dans les travaux de la convention chargée d'élaborer un projet de Constitution pour l'Europe s'est ensuite porté sur la mention des racines religieuses de l'Europe dans le préambule de la Charte puis de la «Constitution». Finalement une formule neutre est privilégiée dans la Charte dont le préambule fait simplement référence au «patrimoine spirituel et moral » de l'Europe, une référence plus franche aux «héritages culturels, religieux et humanistes de l'Europe " étant finalement adoptée dans le préambule du traité sur l'Union européenne. Dans le corps des traités, l'article 17 \& 1 TFUE constitue une véritable constitutionnalisation de la Déclaration $\mathrm{n}^{\circ} 11$ annexée au traité d'Amsterdam; il prévoit que «l'Union respecte et ne préjuge pas du statut dont bénéficient, en vertu du droit national, les églises et les associations ou communautés religieuses dans les États membres». Ces débats, souvent houleux, illustrent parfaitement les diverses sensibilités des pays européens sur cette question et leurs grandes divergences sur la question.

Cette diversité est aussi marquante dans le cadre de l'Europe élargie des pays membres du Conseil de l'Europe dont la proximité avec une religion sera appréciée à l'aune de la Convention européenne des droits de l'homme et de la jurisprudence de la Cour européenne des droits de l'homme. Si tous les États contractants s'engagent à respecter la liberté de pensée, de conscience et de religion et à ne pas discriminer selon la religion, les relations entre l'État et les religions n'en sont pas moins hétéroclites. Pour plusieurs d'entre eux, une relation privilégiée avec la religion dominante parfois érigée en religion d'État est constitutionnellement établie sans être aucunement inconventionnelle. Comme le rappelait la Commission européenne des droits de l'homme, «un système d'église d'État ne peut pas en lui-même être considéré comme violant l'article 9 de la Convention» (liberté de religion). Elle souligne qu'un «tel système existe dans le cadre de plusieurs États contractants et existait déjà quand la Convention a été rédigée et quand ces États sont devenus parties ${ }^{11} »$. À l'époque comme aujourd'hui, Dieu et la religion sont encore présents dans la Constitution de nombre d'États parties à la Convention

international sur les droits civils et politiques, mais ont formulé des réserves établissant la suprématie du droit musulman: Bahreïn, Mauritanie, Qatar, Maldives.

11. Rapport 9 mai 1989, Darby c. Suède, § 45. 
européenne, parfois dans le préambule ${ }^{12}$, quelquefois dans le texte même de la Constitution ${ }^{13}$, souvent dans les deux, préambule et corps du texte ${ }^{14}$. Cet engagement fort à respecter l'identité religieuse d'une nation est quelquefois aussi magnifié par les cérémonies de prestation de serment des plus hautes autorités de l'État ${ }^{15}$. Ainsi, même si dans certains de ces États les réformes vont dans le sens d'une plus grande neutralité affichée ${ }^{16}$, l'identité religieuse de nombre d'États européens demeure forte et, parfois implicitement, valide un traitement préférentiel accordé à la religion historiquement dominante.

12. Loi fondamentale d'Allemagne du 23 mai 1949; Constitution de Pologne du 2 avril 1997; Constitution de la Suisse du 18 avril 1999 proclamée «Au nom de Dieu Tout-Puissant! »; Constitution de l'Albanie du 21 octobre 1998 (« Nous, peuple albanais, fier et conscient de notre histoire tournée vers le futur dans la foi en Dieu ou en d'autres valeurs universelles [...]»). Sans oublier « l'Être suprême» du préambule de la Déclaration française de 1789!

13. Art. 9 de la Constitution de Monaco ( «La religion catholique, apostolique et romaine est religion d'État); art. 18 \& 1 de la Constitution de la République d'Arménie qui consacre la séparation en reconnaissant un rôle particulier à «la Sainte Église apostolique arménienne en tant qu'Église nationale»; art. $11 \S 3$ de la Constitution d'Andorre sur les garanties accordées à l'Église catholique. Constitution de Norvège du 17 mai 1814, art. 2, 3, 9, 16 (l'Église évangélique luthérienne demeure l'Église d'État norvégienne et est «soutenue comme telle»); Constitution du Danemark du 5 juin 1953, art. 4 et 6 ; art. $13 \S 3$ de la Constitution de la Bulgarie ( La religion traditionnelle en République de Bulgarie est le culte orthodoxe»); Constitution de Malte du 21 septembre 1964, art. 2.

14. Par ex. le préambule de la Constitution du $1^{\text {er }}$ juillet 1937 de la République d'Irlande introduit ce texte «au nom de la Très Sainte Trinité» et rappelle «nos obligations envers notre Seigneur Jésus Christ». L'article 6 de la Constitution prévoit que «tous les pouvoirs [...] émanent, après Dieu, du peuple». Le délit de blasphème prévu à l'article 40 \& $61 \mathrm{i}$ a été abrogé par référendum le 26 octobre 2018, mais l'article 44 \& 1 dispose que «L'État reconnaît que l'hommage de l'adoration publique est dû au Dieu Tout Puissant. Son nom est révéré et on doit respecter et honorer la religion». Le texte conclut «à la gloire de Dieu et de l'Irlande». L'originalité de l'Irlande, État chrétien assumé, est d'être non confessionnel puisque le même article $44 \S 2$ interdit à l'État de doter une religion. La Constitution grecque du 9 juin 1975 est proclamée, dans son préambule, «au nom de la Trinité Sainte, consubstantielle et indivisible» et l'article 3 consacre «l'Église orthodoxe orientale du Christ» comme «religion dominante» placée sous l'autorité de «Notre Seigneur Jésus Christ» et est «indissolublement unie, quant au dogme, à la Grande Église de Constantinople» ( $3 \S 1$ ); par ailleurs «le texte des Saintes Écritures reste inaltérable» (3 § 3); enfin l'article 13 interdit le prosélytisme. Constitution du Liechtenstein du 5 octobre 1921, préambule et art. 37 \& 2.

15. Par ex. la prestation de serment du monarque britannique dont le texte et la gestuelle (sur la Bible du Roi Jacques, présentation de l'eucharistie...) sont empreints de religiosité, celle du roi de Norvège prévue par l'article 9 de la Constitution, celle du président de Malte et de son Premier ministre (art. 50 et 89 de la Constitution); pour les fonctionnaires, ministres ou parlementaires, la référence à Dieu est devenue facultative (Allemagne, Suisse).

16. Comme en Suède où les dispositions transitoires de la Constitution du 28 février 1974 relatives à l'Église de Suède ont été abrogées par une loi issue d'un accord entre les autorités séculaires et ecclésiastiques pour sortir du statut d'Église d'État conféré à l'Église luthérienne de Suède, le nouveau régime entrant en vigueur au $1^{\text {er }}$ janvier 2000. Cette Église conserve cependant de nombreux avantages. 


\subsection{UN TRAITEMENT PRÉFÉRENTIEL DIFFUS}

Outre ces proclamations ou formulations générales, l'affichage de liens étroits de l'État avec la religion dominante, vecteur d'une identité à la fois religieuse mais aussi culturelle et historique, emprunte d'autres voies. Aux prestations de serment des plus hautes autorités de l'État s'ajoutent parfois des formules rituelles d'accès à certaines professions ${ }^{17}$ ou des contraintes civiques potentiellement discriminatoires ${ }^{18}$. Le soutien plus ou moins manifeste à l'Église représentative de la religion dominante s'exprime au travers de politiques publiques. Tel peut être le cas, par exemple des dispositions particulières relatives au financement des cultes faisant apparaître un traitement plus favorable pour la religion historique ${ }^{19}$, des systèmes concordataires ou de conventionnement jouant un rôle important par exemple dans le statut des ministres des cultes concernés ${ }^{20}$, l'entretien ou la gestion des lieux de culte $^{21}$, les cours de religion obligatoires 22 pour ne citer que les exemples les plus connus mais reposant sur des mécaniques complexes, parfois opaques. La démonstration de l'attachement d'un État à la religion dominante peut aussi être physique par la présence dans des lieux ou établissements publics de symboles de cette religion. Par exemple en Italie, alors même que la Constitution a endossé les habits de la laïcité-neutralitée ${ }^{23}$, les crucifix doivent

17. À Saint-Marin pour les députés en vertu d'une loi électorale de 1955 obligeant à prêter serment «sur les saints Évangiles» et en Grèce pour les témoins en vertu du Code de procédure pénale, ceux-ci pouvant néanmoins se déclarer hétérodoxes et opter pour une prestation de serment plus neutre mais obligeant le témoin à afficher ses convictions. Ces dispositions inconventionnelles ont été abrogées suite aux interventions de la Cour européenne (CEDH, Gde ch., 18 févr. 1999, Buscarini et a. c. Saint-Marin; CEDH, 8 janv. 2013, Dimitras et a. c. Grèce).

18. L'obligation par ex. de faire figurer sa religion sur les cartes d'identité comme en Turquie, elle aussi sanctionnée par la Cour européenne (CEDH, 2 févr. 2010, Sinan Işık c. Turquie).

19. V. le dossier Le financement public des cultes dans une société sécularisée, coord. par F. Messner, Revue du droit des religions, $\mathrm{n}^{\circ}$ 1, 2016.

20. V. le dossier Quel statut pour les ministres du culte? coord. par F. MESSNER, Revue du droit des religions, $\mathrm{n}^{\circ} 8,2019$, spéc. F. MESSNER, «Le statut des ministres du culte dans les États de l'Union européenne», p. 43-58.

21. V. le dossier Les enjeux contemporains du patrimoine culturel et religieux coord. par A. ForNEROD, Revue du droit des religions, n ${ }^{\circ}$ 3, 2017 qui fait paradoxalement ressortir le soutien aux lieux de culte traditionnels notamment de l'Église catholique dans les États se réclamant de la laïcité-neutralité (France, Belgique) et les problèmes rencontrés dans les États plus ouvertement proches de la religion dominante comme la Suède.

22. G. GonZAlez, «Le droit à l'instruction religieuse dans la Convention EDH», Revue du droit local $\mathrm{n}^{\circ} 81$, Numéro spécial sur l'enseignement religieux, 2017, p. 25-28; «Le droit à l'instruction au sens de la Convention européenne des droits de l'homme », RFDA 2010, p. 1003-1010.

23. L'article 7 de la Constitution de 1948 prévoit: «l'État et l'Église catholique sont, chacun dans son ordre, indépendants et souverains. Leurs rapports sont réglementés par les pactes 
être accrochés dans les salles de classe en vertu d'un décret royal $\mathrm{n}^{\circ} 965$ du 30 avril 1924 dont l'article 118 prévoit que «chaque établissement scolaire doit avoir le drapeau national, chaque salle de classe l'image du crucifix et le portrait du roi », obligation confirmée s'agissant du crucifix par la suite ${ }^{24}$. Cette obligation a été contestée devant la Cour de Strasbourg et les arguments présentés attestent de l'attachement à l'identité religieuse de l'État quand bien même celui-ci aurait-il pris le virage de la laïcité. Avant d'en venir à la solution adoptée par la Cour européenne (partie 2), il est intéressant d'examiner le contexte dans lequel l'affaire est jugée par la Grande Chambre de cette juridiction après que la chambre ait conclu le 3 novembre 2009, à l'unanimité, que la présence obligatoire des crucifix dans les salles de classe violait l'article 2 du Protocole 1 (droit à l'instruction) examiné conjointement avec l'article 9 de la Convention garantissant, ici, la liberté de pensée. La Grande Chambre se livre à une étude de droit comparé qu'avait négligée la chambre. Elle relève que

«la présence de symboles religieux dans les écoles publiques n'est expressément interdite que dans un petit nombre d'États membres: en ex-République yougoslave de Macédoine, en France (sauf en Alsace et en Moselle) et en Géorgie [...] n'est expressément prévue - outre en Italie - que dans quelques États membres: en Autriche, dans certains Länder d'Allemagne et communes suisses, et en Pologne [...] l'on trouve de tels symboles dans les écoles publiques de certains des États membres où la question n'est pas spécifiquement réglementée tels que l'Espagne, la Grèce, l'Irlande, Malte, Saint-Marin et la Roumanie ${ }^{25} »$.

Dans son argumentaire, le Gouvernement italien souligne les diverses significations des symboles religieux, la croix pouvant être perçue «non seulement comme un symbole religieux, mais aussi comme un symbole culturel et identitaire». Sa présence sur les murs des écoles serait «l'expression d'une

du Latran. Les modifications des pactes, acceptées par les deux parties, n'exigent pas de procédure de révision constitutionnelle.» Or, selon le protocole additionnel au nouveau concordat du 18 février 1984, ratifié par la loi no 121 du 25 mars 1985, le principe posé par les pactes du Latran selon lequel la religion catholique est la seule religion de l'État n'est plus en vigueur, la valeur constitutionnelle du principe de laicité étant affirmée par le Conseil constitutionnel (arrêt no 203 du 12 avril 1989).

24. Décret royal $n^{\circ} 1297$ du 26 avril 1928, portant approbation du règlement général des services d'enseignement primaire (approvazione del regolamento generale sui servizi dell'istruzione elementare) dont l'article 119 précise que le crucifix figure parmi les « équipements et matériels nécessaires aux salles de classe des écoles»; obligation validée par le Conseil d'État (13 févr. 2006, n 556 - par une ordonnance du 15 décembre 2004, nº 389 la Cour constitutionnelle se déclare incompétente) et par une directive no 2666 du 3 octobre 2002 du ministre de l'Instruction, de l'Université et de la Recherche.

25. CEDH, Gde ch., 18 mars 2011, Lautsi et a. c. Italie, §§ 26-27. 
"particularité nationale", caractérisée notamment par des rapports étroits entre l'État, le peuple et le catholicisme, qui s'expliquent par l'évolution historique, culturelle et territoriale de l'Italie ainsi que par un enracinement profond et ancien des valeurs du catholicisme» et son maintien reviendrait «à préserver une tradition séculaire ${ }^{26}$ ». D'après lui, «la présence du crucifix dans les salles de classe contribue légitimement à faire comprendre aux enfants la communauté nationale dans laquelle ils ont vocation à s'intégrer $^{27} »$. Cette affaire a suscité une avalanche de tierces interventions faisant valoir, soit des arguments en faveur des requérants émanant exclusivement d'organisations non gouvernementales, soit en faveur du Gouvernement émanant d'autres États parties. Ainsi, les gouvernements de l'Arménie, de la Bulgarie, de Chypre, de la Fédération de Russie, de la Grèce, de la Lituanie, de Malte et de la République de Saint-Marin soulignent dans une tierce intervention commune que "plus de la moitié de la population européenne vit dans un pays non laique [...] inévitablement, des symboles de l'État sont présents dans les lieux où l'éducation publique est dispensée, et que nombre de ces symboles ont une origine religieuse, la croix - qui serait autant un symbole national que religieux - n'en étant que l'exemple le plus visible». Ils ajoutent que «dans les États européens non laiques, la présence de symboles religieux dans l'espace public est largement tolérée par les adeptes de la laïcité, comme faisant partie de l'identité nationale ${ }^{28} »$. Dans une intervention séparée, la Principauté de Monaco précise que «le crucifix est un "symbole passif", que l'on trouve sur les armoiries ou drapeaux de nombreux États et qui en l'espèce témoigne d'une identité nationale enracinée dans l'histoire ${ }^{29}$ » et la Roumanie souligne l'absence de consensus européen qui doit laisser aux États une «large marge d'appréciation» sur cette question sensible ${ }^{30}$. Cette affaire illustre combien cet affichage de l'identité religieuse d'un État peut susciter de tensions et d'inquiétudes de la part de ceux qui, tout en se réclamant de l'identité nationale, ne veulent pas s'en voir imposer l'héritage culturel et/ou religieux. Quoi qu'il en soit, et plus généralement, dans une société démocratique, un État de droit, les effets potentiellement ou effectivement réducteurs des droits d'autrui, discriminatoires, doivent absolument être gommés.

26. Ibid., \& 36 .

27. Ibid., \& 39 .

28. Ibid., \& 47 .

29. Ibid., \& 48 .

30. Ibid., \& 49 . 


\section{L'IDENTITÉ RELIGIEUSE ENCADRÉE}

La jurisprudence de la Cour européenne des droits de l'homme fournit aujourd'hui un panel des conséquences acceptables (2.1) ou néfastes (2.2) de la position dominante reconnue à une religion dans un État partie.

\subsection{LA COMPLICITÉ ÉTATIQUE DÉMASQUÉE DANS L'ABUS DE POSITION DOMINANTE D'UNE ÉGLISE}

Comme cela a été souligné, la Convention européenne des droits de l'homme s'accommode de divers régimes de rapports entre un État et les religions, y compris la consécration de rapports privilégiés avec une religion d'État. Il est néanmoins une limite à ne pas franchir. Si la Cour considère qu'«un parti politique qui s'inspire des valeurs morales imposées par une religion ne saurait être considéré d'emblée comme une formation enfreignant les principes fondamentaux de la démocratie, tels qu'ils ressortent de la Convention ${ }^{31} »$, elle juge nécessaire dans une société démocratique de se prémunir contre l'instauration d'un État théocratique en procédant à la dissolution d'un parti dont le programme est fondé sur un système multijuridique en fonction de la religion et prévoyant l'application de la charia, toutes choses incompatibles avec la notion de société démocratique au sens de la Convention ${ }^{32}$. Ici, l'identité du système conventionnel ne saurait, même aménagée, s'accorder avec un régime fondé sur la théocratie qui «obligerait les individus à obéir non pas à des règles établies par l'État [...] mais à des règles statiques de droit imposées par la religion concernée ${ }^{33} »$. C'est en vertu des mêmes principes d'ailleurs que la Cour européenne protège la forte identité conventionnelle qui s'impose aux États parties et dont ils doivent eux-mêmes être les premiers garants. Dans ce cas aucune dérogation ou tolérance ne peut être admise qui mettrait à mal cette «identité». Ce verrou se drape d'effets extraterritoriaux assurant la garantie des droits indérogeables ${ }^{34}$, notamment en cas d'expulsion ou d'extradition vers

31. CEDH, Gde ch., 13 févr. 2003, Refah Partisi et a. c. Turquie, § 100.

32. Ibid., §§ 117-136; Tout programme ou toute propagande en faveur de l'établissement d'un État théocratique ou de la charia peut entraîner l'application de l'article 17 de la Convention, disposition couperet qui interdit de réclamer l'application des garanties de la Convention: CEDH, déc. 19 juin 2012, Hizb Ut-Tahrir et a. c. Allemagne; déc. 20 juill. 2017, Belkacem c. Belgique.

33. Refah Partisi, précit., § 119.

34. L'effet extraterritorial est ici indirect puisque seul l'État partie à la Convention serait trouvé en violation de la Convention en cas d'expulsion et non le pays destinataire nonpartie à la Convention. 
un pays pratiquant les traitements inhumains et dégradants, la torture ou la peine de mort, en application de préceptes de droit musulman ${ }^{35}$. Le bouclier conventionnel se déploie ainsi pour protéger les victimes potentielles du poids mortifère des traditions ou préceptes religieux issus de la charia encore appliqués par certains des États se réclamant d'un islam constitutionnel.

En dehors de cette situation extrême, le contentieux devant la Cour européenne des droits de l'homme atteste des effets parfois pervers d'un régime d'association étroit entre l'État et une religion dominante. La première partie a mis l'accent sur la situation privilégiée de l'Église orthodoxe en Grèce, situation confortée par la Constitution de ce pays. Il n'est pas anodin de constater que le premier arrêt de la Cour européenne concernant la liberté de religion portait sur la situation difficile faite en Grèce à la minorité des Témoins de Jéhovah. La Cour a clairement posé le conflit de droit généré par certaines situations, identité religieuse de l'État lié à une religion à statut privilégié contre identité des croyants appartenant à une minorité religieuse. Tel est le sens de l'affirmation de principe de la Cour selon laquelle la liberté de pensée, de conscience et de religion «figure, dans sa dimension religieuse, parmi les éléments les plus essentiels de l'identité des croyants et de leur conception de la vie, mais elle est aussi un bien précieux pour les athées, les agnostiques, les sceptiques ou les indifférents ${ }^{36}{ }_{»}$. Dans son opinion concordante, le juge Pettiti souligne que cette liberté « est un droit fondamental et [...] doit pouvoir s'exercer en faveur de toutes les religions et non au profit d'une seule Église, même si celle-ci par tradition historique est Église d'État ou "religion dominante" ». Cette complicité liberticide entre les autorités étatiques et la religion dominante apparaît au cœur d'une autre affaire grecque concernant l'obstacle mis à la libre disposition de lieux de culte par ce même groupement des Témoins de Jéhovah. Ce qui est en cause dans cette affaire ce n'est pas le régime d'autorisation d'ouverture nécessaire pour contrôler le respect des règles d'ordre public, mais l'utilisation arbitraire qui en est faite pour s'opposer à la concurrence que pourrait subir l'Église orthodoxe de Grèce, dont un représentant participe d'ailleurs à la procédure d'autorisation ${ }^{37}$. Le Gouvernement

35. CEDH, 11 juill. 2000, Jabari c. Turquie: risque d'être jugée pour adultère en cas de renvoi en Iran et d'être condamnée à la lapidation; 22 juin 2006, D. c. Turquie: risque de châtiments corporels en cas de renvoi vers l'Iran; Gde ch., 23 mars 2016, F.G. c. Suède: risque de violation des articles 2 et 3 en cas de renvoi de ce converti au christianisme vers l'Iran; 6 juin 2013, M.E. c. France: ici il s'agit de protéger le requérant contre des risques de violences privées contre les coptes d'Égypte devant l'incapacité des autorités locales à assurer leur protection.

36. CEDH, 25 mai 1993, Kokkinakis c. Grèce, § 31.

37. CEDH, 26 sept. 1996, Manoussakis c. Grèce: RTDH 1997, p. 521, obs. G. Gonzalez. 
fait valoir que «pratiquement la totalité de la population est de confession chrétienne orthodoxe, laquelle est étroitement liée à des moments importants de l'histoire de la nation grecque; l'Église orthodoxe préserva la conscience nationale et le patriotisme des Grecs pendant les périodes d'occupation étrangère» et il fustige les groupements minoritaires qualifiés de «sectes ${ }^{38}$. En vain, la Cour soulignant qu'«il ressort du dossier, ainsi que de nombreux cas rapportés par les requérants et non contestés par le Gouvernement, que l'État tend à se servir des potentialités des dispositions susmentionnées de manière à imposer des conditions rigides ou mêmes prohibitives à l'exercice de certains cultes non orthodoxes, notamment celui des témoins de Jéhovah ${ }^{39}$ » qui se voient imposer des sanctions pénales pour la pratique de leur culte collectif, faute d'autorisations, situation par ailleurs dénoncée, sans succès, par le Conseil d'État grec. Dans son opinion concordante, le juge Martens souligne que lorsque «la liberté de religion se trouve en jeu [...] la question est très délicate car des arguments d'ordre public peuvent aisément masquer une intolérance [...] d'autant plus lorsque l'État a une religion officielle». On ne saurait mieux appeler à la vigilance dans ce cas de figure et d'autres exemples attestent, notamment en Grèce ${ }^{40}$ mais aussi dans d'autres pays dotés d'une forte identité religieuse, des risques d'arbitraire étatique tendant à sauvegarder la religion majoritaire ${ }^{41}$, y compris lorsque la religion dominante est menacée dans son intégrité par une fraction schismatique ${ }^{42}$. Les situations

38. Ibid., \& 39 .

39. Ibid., \& 48 .

40. À l'égard des Témoins de Jéhovah mais aussi de la minorité catholique: CEDH, 16 déc. 1997, Église catholique de La Canée c. Grèce.

41. CEDH, 19 nov. 2013, Bogdan Vodă Greek-Catholic Parish v. Romania; 24 mai 2016, Association de solidarité avec les témoins de Jéhovah et a. c. Turquie. En Turquie, la jurisprudence sur les fondations de droit turc, issues du système juridique de l'Empire ottoman et du traité de Lausanne (1923) concernant la protection des anciennes fondations assurant les services publics pour les minorités religieuses, illustre les difficultés des minorités non-musulmanes à préserver leurs édifices, entraînant des constats de violation de l'article 1 Protocole 1 de la Convention (droit des biens): CEDH, 14 déc. 2000 (accord amiable), Institut de prêtres français et a. c. Turquie; 9 janv. 2007, Fener Rum Erkek Lisesi Vakfı c. Turquie; 8 juill. 2008, Fener Rum Patrikliği (Patriarcat oecuménique) c. Turquie; 16 déc. 2008, Yedikule Surp Pırgiç Ermeni Hastanesi Vakfı c. Turquie ( $\left.n^{\circ} 2\right) ; 3$ mars 2009, Bozcaada Kimisis Teodoku Rum Ortodoks Kilisesi Vakfi c. Turquie ( $\left.n^{\circ} 2\right) ; 6$ oct. 2009, Özbek et a. c. Turquie.

42. CEDH, 13 déc. 2001, Église métropolitaine de Bessarabie et a. c. Moldova; 14 juin 2007, Svyato-Mykhaylivska Parafiya v. Ukraine; 15 sept. 2009, Mirolubovs et a. c. Lettonie. Les alévis en Turquie entrent aussi dans ce cadre: Gde ch., 26 avr. 2016, İzzettin Doğan et a. c. Turquie (dans une opinion partiellement dissidente, quatre juges soulignent que «concrètement l'interprétation sunnite de l'Islam s'analyse en Turquie en une "religion d'État" de facto»). 
les plus graves mettent en cause l'obligation positive des États de protéger les religions minoritaires et leurs membres contre les violences plutôt que de tolérer les agressions menées par les partisans (peut-on ici parler de fidèles?) de la religion dominante ${ }^{43}$. La Cour veille aussi à ce que l'État instructeur ne se livre pas à un prosélytisme d'État, abusant ainsi de sa position d'autorité dans un régime d'éducation obligatoire. Si des cours de religion peuvent faire partie des matières obligatoires dans les programmes scolaires, c'est à la condition de ne pas verser dans le confessionnel au profit des dogmes de la religion dominante, sauf à prévoir un régime de dispense aisément accessible et non intrusif dans les convictions des parents ou des enfants eux-mêmes ${ }^{44}$.

\subsection{VADE MECUM D'UNE POSITION DOMINANTE PONDÉRÉE}

Dès lors que les États tireront toutes les leçons de la condamnation de leur complicité démasquée dans les abus de position dominante d'une religion, ils devraient pouvoir poursuivre paisiblement leur politique de proximité avec cette religion tout en respectant les principes de pluralisme et de nondiscrimination à destination des autres religions présentes sur leur territoire et minoritaires. Les exemples ne manquent pas de bonne gestion de telles relations apaisées, dont les États en délicatesse avec la Convention sur la nature de leurs relations avec la religion dominante peuvent s'inspirer. Ainsi la Cour européenne a-t-elle validé le principe de l'impôt ecclésial au profit exclusif de la religion dominante implicitement d'abord ${ }^{45}$, puis plus expressément dans une décision d'irrecevabilité d'une plainte de requérants espagnols se plaignant de ne pouvoir, en tant que membres de l'Église évangélique baptiste, affecter directement une partie de leur impôt au soutien de leur Église, à la différence des contribuables de religion catholique ${ }^{46}$. Selon la Cour, «la liberté de religion n'implique nullement que les Églises ou leurs fidèles doivent se voir accorder un statut fiscal différent de celui des autres contribuables» et en l'absence de consensus européen en matière de financement des cultes, les États jouissent d'une marge d'appréciation pour régler « ces questions [...] étroitement liées

43. CEDH, 6 juill. 2004, 97 membres de la congrégation de Gldani des témoins de Jéhovah et quatre autres c. Géorgie: tabassage de Témoins de Jéhovah, dont des femmes et des enfants, par un groupe de religieux orthodoxes, perpétré avec la complicité passive des autorités policières; 7 oct. 2014, Begheluri et a. c. Géorgie: agressions du même genre avec participation active d'agents de l'État; 24 févr. 2015, Karaahamed c. Bulgarie: troubles devant une mosquée à l'heure de la prière sans intervention de la police.

44. CEDH, Gde ch., 29 juin 2007, Folgero et a. c. Norvège: RTDH 2008, p. 251, obs. G. GonZALEZ; 9 oct. 2007, Zengin c. Turquie; 16 sept. 2014, Mansur Yalçin et a. c. Turquie.

45. Darby c. Suède, précit.

46. CEDH, déc. 14 juin 2001, Alujer Fernandez et Rosa Caballero Garcia c. Espagne. 
à l'histoire et aux traditions de chaque pays». En l'espèce, le «traitement fiscal spécifique dont bénéficie l'Église catholique en Espagne [...] découle des accords conclus le 3 janvier 1979 entre l'Espagne et le Saint-Siège, qui mettent à la charge des deux parties des obligations réciproques: par exemple, l'Église catholique s'engage à mettre au service de la société son patrimoine historique, artistique et documentaire ", la contrepartie étant que «ses locaux de culte bénéficient d'une exonération fiscale». D’une façon générale, «l'obligation faite à une Église de conclure un accord avec l'État afin de pouvoir bénéficier de la rétrocession d'une partie de l'impôt sur le revenu recouvré par l'État n'apparaît pas comme étant une exigence dénuée de fondement ni disproportionnée». Toutefois, la Cour fixe une limite aux conséquences de tels accords qui ne doivent pas constituer un avantage discriminatoire, c'està-dire qu'il doit être possible pour d'autres Églises de conclure un tel accord. Néanmoins, ici, eu égard aux contreparties apportées par l'Église catholique il semble difficile d'imposer à l'État de conclure un accord avec une Église qui n'en offrirait aucune, du point de vue culturel et historique, aucune Église implantée en Espagne n'étant susceptible de rivaliser avec l'Église catholique, ce qui pourrait légitimer implicitement son statut exclusif. En l'espèce, le traitement spécifique dont bénéficie l'Église catholique est d'autant plus acceptable que «la législation fiscale espagnole ne contraint personne à [lui] verser une partie de ses impôts", chaque contribuable pouvant destiner le pourcentage de ses impôts fixé par la loi budgétaire à des fins d'intérêt social ou général, la législation espagnole permettant par ailleurs à toute personne d'effectuer «des dons à l'Église de son choix dans des conditions fiscales avantageuses». On le voit, tout est question d'équilibre et l'avantage procuré peut reposer sur des justifications objectives et raisonnables qui empêchent d'y voir une discrimination. Le droit de l'Union européenne ouvre cependant un autre front. Les exonérations fiscales que le Royaume d'Espagne accorde à l'Église catholique en vertu de l'accord conclu en 1979 avec le Saint-Siège peuvent poser problème lorsque l'Église catholique, en tant qu'autorité responsable d'une école ecclésiastique, souhaite obtenir le remboursement d'un impôt municipal dont elle a dû s'acquitter à l'occasion de travaux de construction effectués sur un bâtiment scolaire utilisé par une congrégation à des fins notamment d'enseignement. Dans ce cas, juge la Cour de Luxembourg, il convient de vérifier si cette «subvention» a un lien avec les activités à caractère strictement religieux, faute de quoi elle pourrait constituer une aide d'État à une activité «économique» interdite ${ }^{47}$. L'État doit donc veiller à ne pas outrepasser l'inégalité acceptable dans ses relations avec la religion dominante.

47. CJUE, 27 juin 2017, n C -74/16, Congregación de Escuelas Pías Provincia Betania. 
L'affaire Lautsi dont il a déjà été question illustre parfaitement cette quête subtile d'un équilibre précaire qui permet d'accepter l'affichage d'un symbole religieux de la religion dominante. La Grande Chambre admet «qu'en prescrivant la présence du crucifix dans les salles de classe des écoles publiques [...], la réglementation donne à la religion majoritaire du pays une visibilité prépondérante dans l'environnement scolaire» ce qui «ne suffit toutefois pas en soi pour caractériser une démarche d'endoctrinement de la part de l'État défendeur ${ }^{48} »$. Comme pour les cours de religion, ce qui s'avère ici déterminant c'est la posture de l'État, prosélytique ou pas. Outre le «caractère passif $^{49} »$ du symbole religieux de la croix,

«les effets de la visibilité accrue que la présence de crucifix donne au christianisme dans l'espace scolaire méritent d'être encore relativisés [...] cette présence n'est pas associée à un enseignement obligatoire du christianisme [...] l'Italie ouvre parallèlement l'espace scolaire à d'autres religions $[\ldots]$ le port par les élèves du voile islamique et d'autres symboles et tenues vestimentaires à connotation religieuse n'est pas prohibé, des aménagements sont prévus pour faciliter la conciliation de la scolarisation et des pratiques religieuses non majoritaires ${ }^{50}$ ».

L'État italien a ainsi réussi sa conversion au pluralisme sans rien renier de la place du christianisme dans son histoire et sa culture.

Tout n'est pourtant pas pour le mieux dans le meilleur des mondes et la vigilance demeure de mise. Plus insidieusement, les États qui ont partie liée avec une religion dominante, de facto ou de jure, s'efforcent de limiter la concurrence en rendant plus difficile l'accès à un statut juridique pour les religions minoritaires, statut nécessaire à la conduite saine des «affaires» d'une Église (défense ou plainte en justice, location ou construction d'immeubles, statut des desservants...). La jurisprudence surabondante sur cette question devant la Cour européenne atteste de ces moyens détournés d'estomper la visibilité des groupes religieux minoritaires ${ }^{51}$. Depuis 2017, la Russie s'est lancée dans une politique de persécution des groupes religieux minoritaires.

48. Précit., § 71 .

49. Ibid., \& 72 .

50. Ibid., \& 74 .

51. Parmi beaucoup: CEDH, Église catholique de La Canée c. Grèce, précit.; Église métropolitaine de Bessarabie c. Moldova, précit.; 5 avr. 2007, Église de Scientologie de Moscou c. Russie; 31 juill. 2008, Religionsgemeinschaft der Zeugen Jehovahs et a. c. Autriche; 10 juin 2010, Témoins de Jéhovah de Moscou c. Russie; 25 sept. 2012, Jehovas Zeugen in Österreich c. Austria; 8 avr. 2014, Magyar Keresztény Mennonita Egyház et a. c. Hongrie; 24 mai 2016, Assoc. de solidarité avec les Témoins de Jéhovah et a. c. Turquie; İzzettin Doğan et a. c. Turquie, précit. 
Les Témoins de Jéhovah notamment sont une organisation interdite depuis une décision de la Cour suprême de Russie du 20 avril 2017 et les autorités s'appuient dans leurs interventions (irruption dans les lieux de culte, saisie des ouvrages, emprisonnement et condamnation de fidèles) sur l'article 282.2.1 du Code pénal russe consacré à la répression des «activités d'une organisation extrémiste ${ }^{52} »$. Ce n'est un secret pour personne que l'Église orthodoxe, proche du pouvoir, exerce une forte influence en Russie ${ }^{53}$. Cette situation perdure alors que la Russie a été condamnée pour violation de la liberté de religion en 2010 par la Cour européenne des droits de l'homme pour avoir procédé à la dissolution de ce même groupement ${ }^{54}$. La leçon n'a pas été entendue. Mais quelque État voyou ne doit pas faire désespérer de tout et il faut prendre en compte, pour rester un peu optimiste, les avancées positives relevées dans la plupart des pays membres du Conseil de l'Europe et parties à la Convention européenne qui assument leur attachement à leur identité religieuse, ce qui est bien normal, dans un climat paisible ou apaisé après avoir corrigé leur comportement dans un sens plus favorable au principe du pluralisme religieux, de la tolérance, de l'État de droit.

52. V. OFPRA, doc. de la DIDR, 2 juill. 2018. Amnesty International rapporte l'extension de cette persécution en Crimée avec des peines d'emprisonnement de six ans uniquement pour expression de sa foi religieuse (communiqué 5 mars 2020). Assemblée parlementaire du Conseil de l'Europe: 12 janv. 2018, doc. 14467 - réponse à question écrite; $1^{\text {er }}$ févr. 2018, déclaration écrite $\mathrm{n}^{\circ} 647$, doc. 14430; 7 févr. 2019, déclaration des corapporteurs de l'Assemblée pour le suivi de la Russie; résolution 2325 (2020), pt 3.8.

53. G. Galstyan, «Le renouveau de l'Église orthodoxe russe après la chute de l'URSS », in N. Guilluy-Sulikashvili (dir.), L'énigme russe: Pouvoir-économie et société, Villeneuve d'Ascq, Presses universitaires du Septentrion, 2012, p. 69-100: http://books.openedition. org/septentrion/46853 [consulté le 17 juin 2020]; A. MONIAK-AzzOPARDI, «Les religions et l'État en Russie. Une relation équivoque», Le Courrier des pays de l'Est, no 2004/5, p. 28-38; La loi sur la liberté de conscience, adoptée le 23 juin 1997, déclare respecter toutes les religions, mais seule l'orthodoxie est qualifiée d' élément inaliénable de l'héritage historique, spirituel et culturel commun de la Russie».

54. Témoins de Jéhovah de Moscou c. Russie, précit. 